\title{
Ablation et gravure laser
}

\author{
D. Débarre, A. Aliouchouche, J. Boulmer, B. Bourguignon*, J.P. Budin et S. Carrez* \\ Institut d'Electronique Fondamentale, URA 22, Bâtiment 220, Université Paris XI, \\ 91405 Orsay cedex, France \\ * Laboratoire de Photophysique Moléculaire du CNRS, Bâtiment 210, Université Paris XI, \\ 91405 Orsay cedex, France
}

\begin{abstract}
RÉSUMÉ : La gravure laser se différencie de l'ablation par l'introduction d'une réaction chimique auxiliaire qui abaisse le seuil de désorption. A l'aide d'exemples sur le silicium et le cuivre, plusieurs types de réaction sont décrites, ainsi que les conséquences sur le substrat. L'importance du choix du laser est également discutée.
\end{abstract}

\section{INTRODUCTION}

Parmi les applications des lasers UV à la modification des surfaces, l'ablation est certainement la plus générale. Elle permet d'enlever à peu près tous les matériaux, même composites, avec une bonne efficacité. Son mécanisme fait intervenir un grand nombre de phénomènes physiques liés aux grandes densités d'énergie laser utilisées. Pour bien des applications les qualités de l'ablation deviennent des défauts : pas de sélectivité chimique, pas de contrôle précis de la profondeur enlevée, température transitoire élevée, non respect des couches sous-jacentes. Le cas des matériaux difficiles à enlever, recouvrant des structures délicates, exemple courant en microélectronique, suffit à comprendre les limites de la méthode: métal réfractaire sur couche de polymère, isolant sur film de $1000 \AA$ de métal, etc... Une méthode adaptée à de tels cas doit donc abaisser le seuil de réaction pour minimiser l'apport d'énergie dans l'échantillon et doit présenter une grande sélectivité chimique pour respecter facilement les autres couches. Une conséquence pratique est de pouvoir augmenter la rentabilité du procédé en utilisant des lasers moins puissants ou corrélativement en traitant des surfaces plus grandes.

La gravure induite par laser utilise pour cela une réaction chimique auxiliaire, le principe étant de faire réagir, spontanément ou à l'aide d'un laser, un gaz sur la surface à traiter et de créer ainsi un nouveau composé chimique, plus volatil que le matériau de départ. La variété des procédés repose sur la manière de créer ce composé, choisie en sorte que la désorption ne soit pas spontanée pour permettre le contrôle du procédé par le laser. En raccourci c'est l'ablation d'une couche superficielle fragilisée par une réaction chimique préalable. Du fait de l'introduction de composés supplémentaires, cette méthode n'est pas générale, et par exemple ne convient pas en cas d'utilisation chimique des produits éjectés pour un dépôt sur un autre substrat. Nous passerons en revue les principaux types de réaction du gaz avec la surface amenant la formation du composé cherché ainsi que les effets secondaires pouvant subsister dans le substrat après désorption par le laser. Les exemples qui seront utilisés concernent les matériaux comme le cuivre et le silicium, liés à des applications en microélectronique. Ce secteur est en effet à l'origine de nombreux développements en photochimie de surface. 
2. ADSORPTION : gravure du silicium par le chlore.

Dans ce cas c'est la chimisorption dissociative du chlore sur le silicium qui est à l'origine de la baisse de l'énergie nécessaire pour la gravure. Cette baisse s'explique d'une part par la diminution de l'énergie de surface lors de l'occupation des liaisons pendantes. D'autre part, cette nouvelle liaison étant conservée lors de la désorption, son énergie va participer favorablement au bilan d'énergie de la gravure. Sur $\mathrm{Si}(100), \mathrm{Cl}_{2}$ s'adsorbe à raison d'un $\mathrm{Cl}$ pour un Si [1]; sur Si(111), le nombre de $\mathrm{Cl}$ peut-être supérieur au nombre de Si [2]. La sélectivité chimique est liée à l'adsorption différentielle sur les divers matériaux et à la nature des liaisons à remplacer. C'est ainsi que l'oxygène inhibe la gravure du silicium et que la silice n'est pas gravée par cette méthode. L'activation de la désorption passe par une excitation et une fragilisation des liaisons restantes avec le substrat, basculant une part de l'énergie en énergie de désorption. Une activation par excitation électronique nécessite l'excitation simultanée de plusieurs liaisons. Sa probabilité est donc quasi nulle. L'activation thermique n'est significative que si les liaisons retenant l'atome sont rendues suffisamment labiles pour permettre soit une substitution des atomes du substrat par des adsorbats soit une cassure simple pour former des radicaux. Son efficacité augmente avec la température

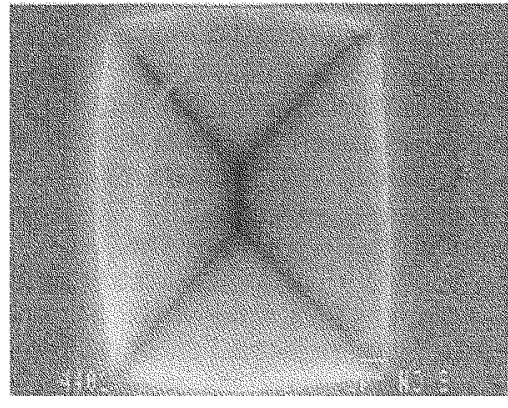

figure 1 : Gravure laser du Si (100) par $\mathrm{Cl}_{2}$ en dessous du seuil de fusion. La tache laser est circulaire et l'élévation de température est inférieure à $500^{\circ} \mathrm{C}$. Les plans révélés sont (111). mais le processus change de nature également. Le produit de désorption spontanée est essentiellement $\mathrm{SiCl}_{4}$ au dessous de $500 \mathrm{~K}$. L'énergie d'activation liée à la désorption de $\mathrm{SiCl}_{2}$ permet à ce produit de devenir prépondérant au dessus de $600 \mathrm{~K}$, $\mathrm{SiCl}$ n'apparaissant qu'au dessus de $900 \mathrm{~K}$ [3]. A température modérée, la vitesse de désorption est limitée par la vitesse de substitution et donc par le nombre de liaisons à substituer ou à briser. Le plan (111), où un atome est lié par une liaison de surface et trois liaisons avec le substrat, se gravera ainsi plus lentement que le plan (100) où un atome est retenu par deux liaisons de surface et deux liaisons avec le substrat. En pratique, partant d'un plan cristallin quelconque, la gravure par un flux laser peu intense révèle les faces les plus difficiles à graver qui imposent leur vitesse (fig. 1). La gravure est alors très peu efficace et pas verticale.

Une gravure non sélective en orientation cristalline passe par la création d'un

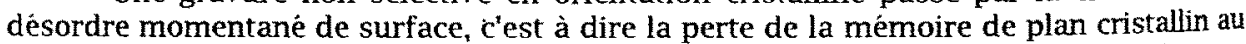
moment de la désorption. Quant à l'efficacité de désorption, elle augmente rapidement avec la température atteinte à la surface; la cassure des liaisons est plus efficace que la substitution. La fusion de la surface correspond à ces deux limites. La rapidité et l'efficacité du processus de désorption en ce cas empêche les adsorbats de se réordonner; $\mathrm{SiCl}$ devient alors un produit essentiel de la gravure avec $\mathrm{SiCl}_{2}$ dont la part est sous estimée dans la plupart des travaux 14,5$]$. En pratique, pour ne pas trop perturber les couches sous-jacentes, la fusion est transitoire pour limiter la quantité d'énergie déposée dans l'échantillon. Le rôle de la longueur d'onde dans le processus fait l'objet de mesures divergentes dans la littérature, alors que l'influence de la phase gazeuse n'y est pas toujours bien dissociée. En contrôlant l'apparition de la fusion par le changement transitoire de réflectivité, nous avons montré que la désorption sous vide n'est pas lice à la longueur d'onde mais à l'apparition de la fusion de la surface [6]. Quand la fusion est complète $\left(\sim 0,5 \mathrm{~J} / \mathrm{cm}^{2}\right.$ pour $10 \mathrm{~ns}$ à $\left.308 \mathrm{~nm}\right)$, la désorption sature et ne dépend plus de l'énergie déposée (voir fig. 3 courbe a). Cet effet de saturation autorise dans les applications, quelques inhomogénéités de faisceau, mais des dégâts apparaissent si on atteint le seuil de dommage du silicium pur $\left(\sim 1 \mathrm{~J} / \mathrm{cm}^{2}\right)$.

La sélectivite chimique est assurée par la chimisorption préalable. Le nombre de 
sites d'adsorption sur la surface limite par ailleurs l'efficacité de gravure et permet un contrôle précis de la profondeur gravée. Comme il suffit d'adsorber une monocouche entre deux tirs laser, la réaction peut avoir lieu sous pression réduite ce qui autorise des mesures de contrôle in-situ et en temps réel. L'efficacité de gravure est dans ces conditions de $0,6 \mathrm{~A} / \mathrm{tir}$, correspondant environ à une demi-monocouche de silicium. C'est moins que ce que le nombre de sites potentiels d'adsorption sur la surface pourrait laisser espérer. On peut s'interroger sur l'origine de cet écart, la désorption non réactive de chlore atomique, inefficace en terme de gravure, n'étant pas suffisante pour l'expliquer [5]. Si l'on arrête l'apport de chlore et donc l'adsorption tout en continuant l'irradiation laser, l'efficacité de désorption ne s'annule pas au tir suivant mais vaut environ $30 \%$ de la valeur au tir précédent. Il en est de même pour les tirs suivants [7]. Le chlore n'est donc pas entièrement désorbé au premier tir laser et il résiste tout aussi efficacement aux tirs suivants. Une analyse Auger et SIMS nous a montré que ces atomes sont essentiellement localisés sur la surface, mais qu'une partie d'entre eux est incorporée dans les premières couches. La barrière de diffusion du chlore dans le silicium cristallin étant de $13 \mathrm{eV}$ [8], cette contamination ne peut s'expliquer que par une incorporation transitoire plus importante dans la phase liquide. En soustrayant une partie des adsorbats, cette incorporation transitoire diminue l'efficacité de gravure et explique le maintien du chlore sur la surface [9].

Le nuage dense de produits désorbés, contenant très peu d'ions, forme une zone dite de Knudsen à grande fréquence de collisions. Celles-ci induisent un réarrangement des vitesses, la nouvelle distribution étant caractérisée par une répartition étroite, centrée le long de la normale à la surface et par une accélération dans cette direction [10]. D'après nos mesures, l'énergie cinétique peut atteindre $10000 \mathrm{~K}$ et, contrariant une possible justification de l'efficacité de désorption inférieure à la monocouche, il n'y a pas en retour de redépôt stable sur la surface. En limitant l'apport de chlore, la densité des produits est suffisamment faible pour éliminer les collisions; l'énergie de translation correspond alors à la température de fusion du silicium $(1680 \mathrm{~K})$ [7].

\section{ADSORPTION ET DIFFUSION : gravure du cuivre [11].}

Dans l'exemple précédent, la réaction chimique, restreinte à la seule surface de la couche, permet un contrôle fin de la profondeur gravée mais limite la vitesse de gravure. Il est des cas où l'agent gravant peut diffuser dans la couche. En se substituant aux liaisons existantes, ces atomes forment avec le matériau de départ un composé, de propriétés optiques et thermiques différentes et qu'on souhaite plus volatil. La diffusion dépend de la qualité cristalline de la couche, mais aussi de la nature chimique des partenaires. Dans le cas de la gravure du cuivre, nous avons mesuré par exemple des vitesses de diffusion plus grandes d'un ordre de grandeur si le chlore est apporté par $\mathrm{Cl}_{2}$ plutôt que par $\mathrm{CCl}_{4}$. Cette diffusion spontanée est accélérée par l'augmentation de la pression et par l'élévation de température induite par le laser ce qui permet de contrôler spatialement et temporellement la réaction.

Toujours dans le cas du cuivre, la vitesse d'ablation de ce composé peut atteindre des valeurs élevées $(20 \mathrm{~A} / \mathrm{tir}$ avec $\mathrm{CCl}_{4}, 10$ fois plus avec $\mathrm{Cl}_{2}$ ). Elle est précédée par une période d'incubation (fig. 2) due à une passivation par la présence d'oxyde en surface. Le faible seuil d'ablation du composé formé $\left(\sim 0,1 \mathrm{~J} / \mathrm{cm}^{2}\right)$ tient à son manque de cohésion et à sa forte absorption optique. Sa composition et sa structure sont encore

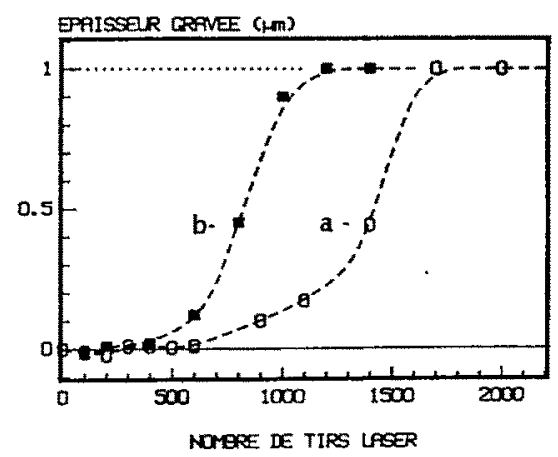

figure 2 : Gravure laser d'un film de cuivre $(1 \mu \mathrm{m})$ pour deux pressions de $\mathrm{CCl}_{4}$ (a - 55 mbar, b - 130 mbar). La présence d'oxydes en surface inhibe d'abord la gravure. Quand la diffusion de $\mathrm{Cl}$ en volume est suffisante, l'efficacité de gravure atteint $20 \mathrm{~A} / \mathrm{tir}$. L'énergie du laser $\left(150 \mathrm{~mJ} / \mathrm{cm}^{2}\right)$ est insuffisante pour graver Si, la gravure s'arrête à l'interface Cu-Si. 
difficiles à préciser du fait de son hétérogénéité. Les études sur la diffusion spontanée [12] montrent que le chlore se substitue au cuivre pour former un composé de type $\mathrm{CuCl}_{\mathrm{X}}(\mathrm{x}<1)$. Les produits éjectés forment un nuage dense mais sans interaction plasma avec la surface aux énergies laser utilisées $\left(\sim 0,15 \mathrm{~J} / \mathrm{cm}^{2}\right)$. La sélectivité repose sur la différence de diffusion dans les différents matériaux et sur l'énergie nécessaire. Il est ainsi aisé de graver des structures sur un film de $\mathrm{Cu} / \mathrm{Si}$ sans dommage pour la sous couche de Si en utilisant un masque de chrome de $400 \AA$ d'épaisseur.

Un inconvénient, lié à la diffusion, est la pollution résiduelle en fin de gravure. Bien qu'elle puisse être contrôlée par le choix de l'agent gravant, elle rend plus délicate la gravure partielle du substrat. Les diffusions spontanée et induite en présence de $\mathrm{Cl}_{2}$ sont si importantes ( $\mathrm{qq} \mu \mathrm{m}$ ) qu'il est difficile, voire impossible, de contrôler la réaction et la pollution résiduelle. Malgré une vitesse de gravure moindre, due à une diffusion moindre $(\sim 500 \AA)$, nos résultats démontrent que, d'un point de vue technologique, il est préfërable d'utiliser un gaz moins réactif comme $\mathrm{CCl}_{4}$.

\section{INFLUENCE DE LA PHASE GAZEUSE : gravure sélective en dopage du silicium.}

Si les espèces réactives possèdent une énergie interne ou cinétique, celle-ci, en participant au bilan énergétique de la désorption, va en augmenter l'efficacité. Cette énergie peut être apportée thermiquement ou par un jet supersonique mais également par interaction laser avec le gaz. La photodissociation du chlore à $308 \mathrm{~nm}$ par exemple confère $0,7 \mathrm{eV}$ à chacun des atomes produits. Cette energie est suffisante pour créer une réaction spontanée à la surface, mais les collisions dans le gaz la convertissent rapidement en élévation de température. Une forte pression de chlore est néanmoins nécessaire pour créer une densité importante de radicaux à la surface et empêcher leur diffusion.

L'adsorption est favorisée par la présence de porteurs libres en surface soit créés par le laser [13], soit dus à un niveau de dopage approprié [14]. On peut penser que l'affinité électronique du chlore joue un rôle important dans ce processus mais des travaux complémentaires restent nécessaires.

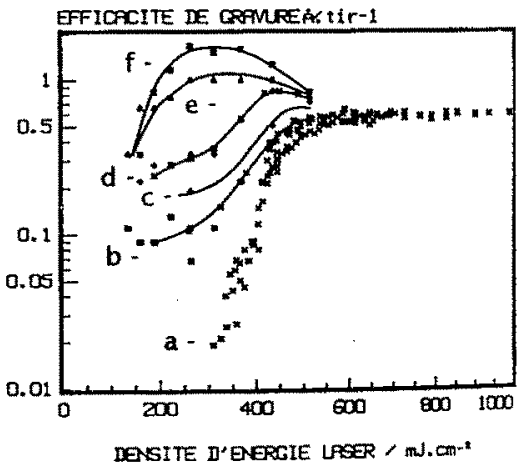

figure 3 : Efficacité de gravure de $\mathrm{Si} / \mathrm{Cl}_{2}$ en fonction de l'énergie du laser $(308 \mathrm{~nm})$ pour différents niveaux de dopage. La pression statique de $\mathrm{Cl}_{2}$ est 130 mbar sauf pour (a) où elle est nuile, $\mathrm{Cl}_{2}$ étant déposé sur la surface avant chaque tir. Dopage : a \& b- $310^{15} \mathrm{c}$. $310^{18}, \mathrm{~d}-110^{20}, \mathrm{e}-310^{20}, \mathrm{f} \cdot 210^{21}$ atómes $/ \mathrm{cm}^{3}$.

Dans une étude sur la gravure en fonction du niveau de dopage, nous avons montré que si l'efficacité de désorption est ainsi améliorée par la création de radicaux dans une vapeur à pression élevée $(\sim 130 \mathrm{mbar})$, elle s'améliore toujours nettement avec la température induite par le laser. En approchant le seuil de fusion elle atteint un maximum quí peut dépasser la valeur atteinte lors de la fusion (fig. 3). Dès que la fusion apparaît les effets électriques sont masqués et le cycle adsorption - désorption est essentiellement limité par les effets thermiques et l'incorporation transitoire du chlore dans la couche en fusion. Les problèmes d'orientation cristalline ne disparaissent là encore que si le bilan énergétique est nettement excédentaire. La sélectivité chimique est conservée car la réaction repose toujours sur l'établissement de liaisons chimiques spécifiques.

\section{CONSÉQUENCES SUR LA COUCHE GRAVÉE}

Faire diffuser des atomes dans la couche pour favoriser la gravure modifie évidemment le matériau et laisse une pollution résiduelle. Même dans le cas où le processus de gravure ne repose pas sur cette diffusion mais sur une simple adsorption en surface, une incorporation involontaire a lieu sur quelques plans atomiques au 
mieux comme on l'a vu dans le cas du silicium. Ce dernier point résulte de la modification majeure, bien que transitoire, que représente la fusion de la surface sous l'impact laser. Cette fusion, destinée à favoriser la désorption des molécules formées après adsorption du gaz, va conduire à d'autres processus corrélés de désorption et de diffusion. La recristallisation de la couche va également amener son cortège de modifications après chaque tir laser. Les exemples qui suivent isolent quelques conséquences et propriétés particulières dues au traitement laser lors de la gravure.

\subsection{Désorption d'impuretés et nettoyage de surface}

L'analyse Auger des premières monocouches d'un échantillon de silicium, pollué par exposition à l'air, montre une présence importante et caractéristique de carbone et d'oxygène masquant le signal dû au silicium (fig. 4). Sous l'effet des impacts laser succèssifs induisant une fusion transitoire, le carbone et l'oxygène sont désorbés et le signal correspondant au silicium atteint le niveau et le profil caractéristique du silicium propre. L'efficacité et la souplesse de cette méthode de nettoyage de la surface se compare très favorablement au traitement thermique conventionnel nécessaire pour éliminer l'oxygène et surtout le carbone. Ce phénomène de nettoyage intervient également pendant la gravure et permet d'opérer dans des conditions expérimentales peu contraignantes en eliminant continuellement des adsorbats

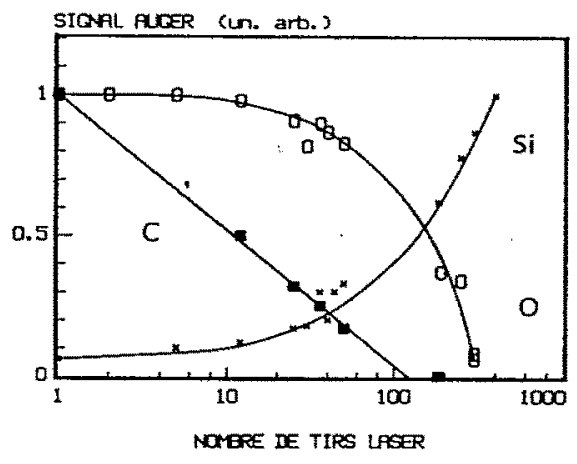

figure 4 : Variation du signal Auger correspondant au carbone, à l'oxygène et au silicium au cours d'un processus de nettoyage sous vide. A chaque tir le laser $\left(600 \mathrm{~mJ} / \mathrm{cm}^{2}\right)$ induit la fusion . indésirables. Les espèces contenues en volume sont également soumises à ce phénomène de désorption dont l'efficacité varie avec la nature des atomes [15].

Il existe un phénomène exactement inverse d'adsorption d'atomes de gaz, favorisée par la présence transitoire de la phase liquide. En quelques tirs laser, on peut incorporer suffisamment d'atomes pour créer des couches très dopées à la surface du silicium [16]. Dans ce cas précis les gaz utilisés s'adsorbent spontanément sur un grand nombre de couches, permettant d'obtenir une incorporation très efficace parallèlement à une forte désorption.

Désorption et incorporation sont en fait une illustration des propriétés de transport des impuretés dans la phase liquide et dont les conséquences sont importantes malgré la brièveté de la fusion.

\subsection{Diffusion des dopants et des impuretés}

Deux paramètres sont à l'origine de ces comportements spectaculaires liés à la fusion transitoire de la surface. D'une part la très grande mobilité des impuretés dans la phase liquide amène la redistribution rapide des concentrations dans la zone fondue. D'autre part la ségrégation au voisinage du front de solidification refoule les impuretés dans la zone fondue. La ségrégation est due à la différence de mobilité et de solubilité dans le solide et le liquide de ces éléments minoritaires. Elle est donc fortement dépendante de la vitesse de solidification. Les profils de concentration qui en résultent vont dépendre alors des propriétés de ces impuretés. Une forte ségrégation ramène les impuretés en surface et favorise la désorption et le nettoyage, une faible ségrégation favorise au contraire l'incorporation et l'uniformisation des concentrations. A l'opposé, une diffusion forte défavorise la gravure, une diffusion faible, l'incorporation.

Dans l'exemple de la figure 5 on présente les profils de concentration en profondeur de plusieurs dopants dans le silicium. Les profils initiaux obtenus par implantation ne sont pas uniformes et on suit leur évolution à différents stades de gravure. On constate que les dopants sont en quelque sorte repoussés dans le substrat 
CONCENTRATION / atomes. $\mathrm{cm}^{-3}$

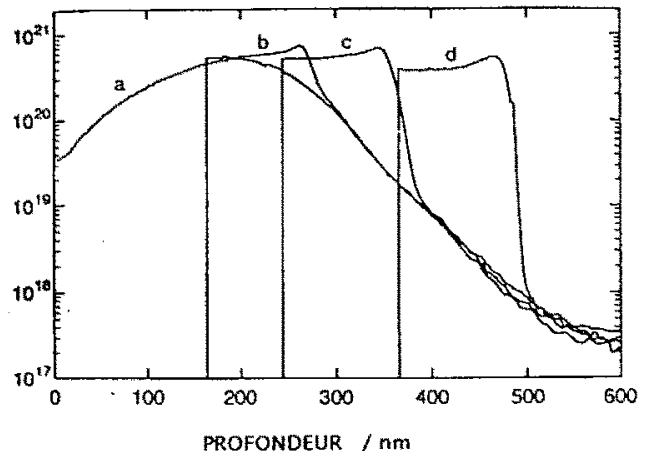

CONCENTRATION / atomes.cm ${ }^{-3}$

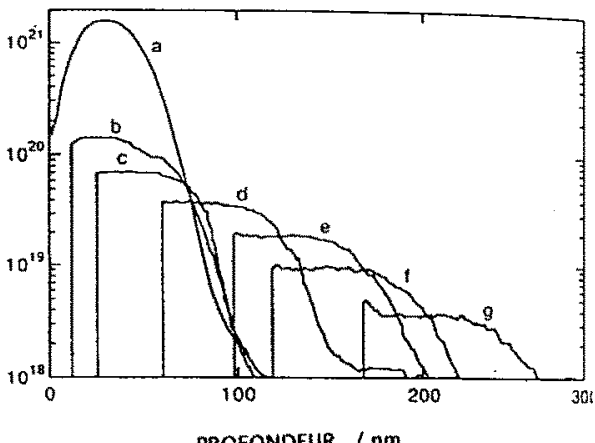

figure 5 : Variation de la concentration en profondeur du bore (1) et de l'antimoine (2) dans Si à différents stades de la gravure de Si. Les dopants sont initialement implantés (a) $(60 \mathrm{keV}$, $610^{15} \mathrm{~cm}^{-2}$ ), les profils en profondeur sont obtenus par analyse SIMS. La surface, représentée par une ligne verticale, est localisée à des profondeurs croissantes au fur et à mesure de la gravure, après (1b) 2900, (Ic) 4000, (1d) 6300, (2b) 200, (2c) 400, (2d) 1000, (2. e) $1600,(2 f) 2000,(2 \mathrm{~g}) 3000$ tirs laser à $308 \mathrm{~nm}$.

au fur et à mesure de la gravure. Ceci indique qu'indépendarmment d'une désorption moins forte (bore) ou plus forte (antimoine) que le silicium lors de la gravure, une partie des dopants est soustrait à la désorption par diffusion dans la zone fondue. La variation de la largeur et de l'uniformité des profils de concentration met en évidence la mobilité des dopants en phase liquide, leur ségrégation au cours de la cristallisation et leur désorption différente. Elle reflète la nature de leur liaison avec l'agent gravant et avec la couche, ainsi que leur limite de solubilité dans la matrice hôte [15]. Les profils et les concentrations ainsi obtenus peuvent atteindre des valeurs inaccessibles par d'autres moyens.

\subsection{Recristallisation}

Le cas du recuit de couches de SiGeC (fig. 6) illustre bien les propriétés de guérison des défauts dans un cristal (ici amorphisé par l'implantation du carbone). A chaque tir, le front de fusion dépasse le fond de la couche défectueuse pour atteindre la structure sous-jacente à partir de laquelle le cristal va se reformer. Pour un tel alliage, malgré les défauts majeurs au départ et la présence d'atomes très dissemblables au point de vue cristallin, nous avons pu montrer que quelques tirs laser suffisent à redistribuer les liaisons et homogénéiser les concentrations. L'énergie utilisée dans ce cas correspond à celle nécessaire pour la gravure, seul manque le gaz réactif. Ainsi, lors de la gravure avec fusion, la couche est continuellement guérie des éventuels défauts pourvu que soient satisfaites les conditions pour une vitesse de solidification adaptée.

\section{"CONIRSSTE" (un. art.)}

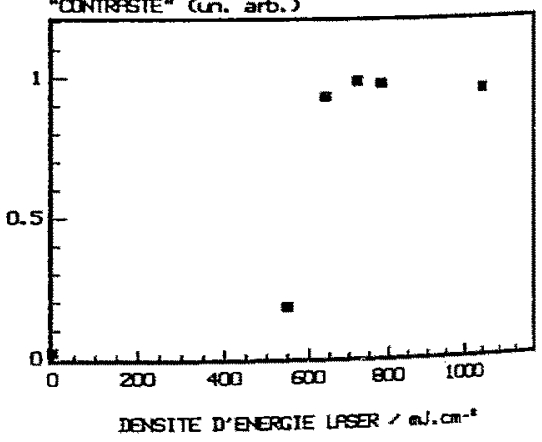

figure 6 : Amélioration de l'état cristallin d'une couche $\mathrm{SiGeC/Si}(900 \mathrm{~A})$ par recuit laser. Analyse du contraste des images de canalisation d'électrons. La couche SiGe/5i initiale est amorphisée par l'implantation du carbone. Le seuil d'amélioration correspond à une profondeur fondue ègale à l'épaisseur de la couche. 


\section{CHOIX DE LA SOURCE}

\subsection{Longueur d'onde et durée}

Dans tout ce qui précède, le laser intervient par un dépôt d'énergie au voisinage de la surface ou une excitation du gaz réactif. Si aucune caractéristique particulière n'a encore été donnét sur ce laser, il n'en reste pas moins que le choix de la longueur d'onde et de la durée de l'impulsion est implicitement contenu dans la description des phénomènes induits. En pratique les lasers à excimère sont les plus utilisés.

L'excitation du gaz qui se produit essentiellement par photodissociation est obtenue efficacement par l'intermédiaire d'une transition directe. Celle-ci se trouve fréquemment dans I'UV ou le VUV mais le choix de la longueur d'onde n'en est pas toujours rendu plus simple : $\mathrm{Cl}_{2}$ est ainsi dissocié à $308 \mathrm{~nm}$ et pas à $248 \mathrm{~nm}$. L'excitation électronique dépend de la nature de la couche. Dans le cas des isolants et des semi-conducteurs à grande bande interdite seule l'irradiation UV permet une excitation directe. Malgré une réflectivité élevée, les métaux ont une épaisseur optique faible pour des énergies inférieures à la fréquence plasma située loin dans I'UV. Toutefois si le matériau se présente sous forme de couche mince, les propriétés optiques peuvent différer sensiblement du matériau massif du fait de l'importance que prennent les effets de surface. De plus des changements radicaux dans le couplage de l'onde incidente avec le solide interviennent avec l'utilisation de fortes densités d'ênergie laser. La production d'une grande densité de porteurs libres, la distortion des bandes d'énergie par le champ de l'onde et par l'élévation de température amènent en général une diminution de la réflectivité et une augmentation considérable de l'absorption.

Or les conditions du dépôt d'énergie dans la couche sont déterminantes. Deux paramètres sont à prendre en compte : l'épaisseur optique et la longueur de diffusion thermique pendant $t$ durée de l'impulsion. Cette longueur varie comme $t^{1 / 2}$. L'énergie nécessaire pour induire la réaction est utilisée de manière optimale si elle est déposée et confinée dans une épaisseur faible. L'échauffement en profondeur de l'échantillon est alors lui-même minimisé. Pour obtenir un pic de température élevé à la surface, il faut apporter l'énergie du laser plus vite qu'elle ne s'évacue par diffusion. De courtes durées d'impulsion sont donc recommandées, typiquement 1 ps dans le cas d'un bon conducteur comme le silicium dont l'épaisseur optique est très faible dans l'UV (45 A). Par voie de conséquence, le gain d'énergie obtenu par augmentation de la durée de l'impulsion, comme dans certains lasers à excimère, n'est pas entièrement transposable en gain de surface traitée à cause de cette diffusion pendant l'impulsion.

La comparaison des deux paramètres change d'aspect dans le cas où la fusion de la surface est atteinte pendant l'impulsion laser. Pour que la reconstruction du cristal soit possible après fusion, il faut que la vitesse du front de solidification soit inférieure à une certaine valeur, propre à chaque matériau $(\sim 15 \mathrm{~m} / \mathrm{s}$ pour Si). Cette vitesse est imposée par le gradient de température dans le matériau à la fin de l'impulsion laser. Dans le cas de $\mathrm{Si}$ un gradient adéquat est obtenu pour des durées d'impulsion supérieures à 2 ns.

\subsection{Homogénéité, cohérence}

Les phénomènes décrits plus haut sont sensibles à l'énergie du faisceau et à sa distribution temporelle, bien que certaines conditions atténuent cette sensibilité. Or il s'agit soit de discerner les processus intervenant dans des conditions différentes d'énergie, soit d'obtenir un traitement homogène de la surface. Il est donc important d'utliser des faisceaux homogènes aussi bien spatialement que temporellement avec un profil spatial plutôt de type plat. Ces exigences sont contradictoires et renvoient au mode de production du faisceau et donc à ses propriétés de cohérence.

D'une manière générale les problèmes liés à la cohérence du faisceau sont souvent ignorés, mais sont une source d'inhomogénéité d'éclairement en créant une figure d'interférence sur la surface. Ces interférences sont dues soit à l'inhomogénéité du faisceau, soit à la diffraction de la lumière par les structures créées ou déjà présentes sur la surface. On retrouve alors cette figure d'interférence imprimée dans l'échantillon. Un faisceau satisfaisant devrait avoir une cohérence nulle. Ces difficultés 
peuvent être surmontées en tenant compte des propriétés particulières de l'échantillon traité. Si la taille de la structure spatiale projetée par le laser est infërieure à la longueur de diffusion de la chaleur pendant l'impulsion, et si celle de la structure temporelle du faisceau est inférieure à la durée de la relaxation de l'excitation électronique dans le solide, ces structures ne seront pas "vues" par l'échantillon. Un fonctionnement en régime multimode peut donc s'envisager s'il y a suffisamment de modes et si pour cela la largeur spectrale du laser est suffisante. C'est le cas pour les lasers à excimère, c'est plus délicat pour les YAG avec 40 ps de temps de cohérence dans l'UV. Dans ce régime multimode il est relativement aisé d'obtenir un profil énergétique spatialement et temporellement acceptable contre une perte modérée d'énergie. L'avantage d'une grande largeur spectrale peut devenir un inconvénient dans le cas de projection de motifs dont la taille approche celle de la longueur d'onde ce qui nécessite alors l'utilisation d'un objectif achromatique très onéreux dans l'UV.

\section{CONCLUSION}

La gravure laser est un cas particulier d'ablation où le seuil a été artificiellement baissé en établissant au préalable des liaisons de substitution à la surface ou dans le volume. L'énergie nécessaire à la désorption est encore suffisante pour induire dans le substrat des perturbations non négligeables. Néanmoins celles-ci peuvent être mises à profit pour guérir des défauts structuraux, libérer des tensions, améliorer les propriétés électriques, modifier les distributions des composants minoritaires (uniformité, proril, concentration). Un des attraits principaux de l'introduction d'une réaction chimique auxiliaire est la sélectivité qu'elle apporte aussi bien en terme de composition chimique, suivant la nature et les propriétés électriques des matériaux, qu'en terme de dosage de la réaction pour le contrôle de la profondeur gravée et l'atténuation des imperfections expérimentales.

\section{Références}

[1]- Craig B.I., Smith P.V., Surf. Sci. 262 (1992) 235.

[2]- Gao Q., Cheng C.C., Chen P.J., Choyke W.J., Yates J.T., J. Chem. Phys. 98 (1993) 8308

[3]- Szabo A., Farrall P.D., Engel T., Surf. Sci. 312 (1994) 284.

[4]- Boulmer J., Bourguignon B., Budin J.P., Débarre D., Appl. Surf. Science 43 (1989) 424

[5]- Bourguignon B., Stoïca M., Dragnea B., Carrez S., Boulmer J., Budin J.P., Débarre D., Aliouchouche A., Surf. Science, soumis.

[6]- Boulmer J., Bourguignon B., Budin J.P., Débarre D., Desmur A., J. Vac. Sci. Technol. A9 (1991) 2923.

[7]- Aliouchouche A., Boulmer J., Bourguignon B., Budin J.P., Débarre D., Desmur A., Appl. Surf. Science 69 (1993) 52.

[8]-Seel M., Bagus P.S., Phys. Rev. B 28 (1983) 2023.

[9]- Boulmer J., Budin J.P., Bourguignon B., Débarre D., Desmur A., in Laser ablation of electronic materials, éditeurs Fogarassy E. et Lazare S. (Elsevier, 1992) pp. 239-254.

[10]- Baller T.S., Kools J.C.S., Dieleman ]., Appl.Surf. Sci. 46 (1990) 292.

[11]- Aliouchouche A., Boulmer J., Budin J.P., Débarre D., Palleau J., "Laser Chemical Etching of Copper Films", Photo-Assisted Processing of Surfaces and Thin Films, E-MRS Spring Meeting, Strasbourg 24-27 mai 1994.

[12]- Sesselmann W., Chuang T.J., Surf.Sci. 176 (1986) 32., Surf.Sci. 176 (1986) 67.

[13]- Kullmer R. and Bäuerle D., Appl. Phys. A 47 (1987) 377.

[14]- Horiike Y., Hayasaka N., Sekine M., Arikado T., Nakase M., Okano H., Appl. Phys. A 44 (1987) 4237.

[15]- Desmur A., Bourguignon B., Boulmer J., Ozenne J.B., Budin J.P., Débarre D., Aliouchouche A., J. Appl. Phys. 76 (1994) 3081.

[16]- Foulon F., Fogarrassy E., Slaoui A., Fuchs C., Unamuno S., Siffert P., Appl. Phys. A 45 (1988) 361. 